\title{
Cryptococcal meningitis in HIV infected: Experience from a North Indian tertiary center
}

\author{
Susheel Kumar, Ajay Wanchu, Arunaloke Chakrabarti ${ }^{1}$, Aman Sharma, Pradeep Bambery, \\ Surjit Singh \\ Departments of Internal Medicine and ${ }^{1}$ Microbiology, PGIMER, Chandigarh, India
}

\begin{abstract}
Background: Cryptococcal meningitis is a common opportunistic infection in Human Immunodeficiency Virus (HIV)-infected individuals. There is little information specifically addressing cryptococcal meningitis in HIV-infected patients from North India. Aims: To determine clinical presentation, hospital course, response to treatment, complications developed, in-hospital mortality, any recurrence of cryptococcal meningitis and reasons of recurrence during follow-up. Settings and Design: A retrospective observational study undertaken in a large tertiary care center. Materials and Methods: Patient's demographic data, presenting clinical symptomatology, physical findings, laboratory parameters, cerebrospinal fluid (CSF) examination findings, side-effects of treatment, development of any complications and hospital outcome were analyzed. During follow-up any recurrence of cryptococcal meningitis, possible reasons of recurrence, type of treatment received, complications developed and outcome was recorded as well. Results: Forty patients diagnosed to have cryptococcal meningitis were analyzed. Twenty-two (55\%) patients had acute/ subacute presentation. Thirty-six (90\%) patients presented with headache and $18(45 \%)$ had altered sensorium. Twenty (50\%) patients had no cells in the CSF. Hypoglycorrhchia was seen in $30(75 \%)$ patients. Cryptococcal meningitis was the first acquired immune deficiency syndrome (AIDS)-defining illness in 30 (75\%) patients. Thirty-five patients developed some adverse effects to amphotericin-B. Thirty-three patients improved with treatment while three patients died. Four patients had recurrence of cryptococcal meningitis within six months of first episode. Non-compliance of fluconazole therapy was the reason for recurrence in all of these patients. Conclusions: Cryptococcal meningitis is a common initial AIDS-defining illness. Acute and/or subacute presentation of cryptococcal meningitis is not uncommon in HIV-infected individuals. An early diagnosis of HIV infection might reduce the incidence of this infection.
\end{abstract}

Key words: Cryptococcal infection, HIV, immunocompromised, India, meningitis, outcome

\section{Introduction}

Human Immunodeficiency Virus (HIV) infection was first reported in India in the state of Tamil Nadu in 1986. ${ }^{[1]}$ This infection has since spread to the entire country. According to official estimates in 2006, adult HIV prevalence was $0.36 \%$ and 2.5 million were infected with the virus. ${ }^{[2]}$ The most common opportunistic infection in HIV patients in India is tuberculosis. ${ }^{[3-6]}$ Cryptococcal meningitis has been reported as the most common opportunistic infection of the central nervous system (CNS) of Indian patients with HIV infection. ${ }^{[3-5]}$ Cryptococcal meningitis caused by environmental fungus Cryptococcus neoformans, is a common opportunistic infection in HIV-infected individuals, particularly in Southeast Asia, Southern and East Africa. ${ }^{[7]}$ It usually infects HIV-infected patients with CD4 count $<100$ cells/ $\mu$ l. These patients present with features of acute, subacute or chronic meningitis or meningoencephalitis. This infection is fatal without treatment. Therefore, rapid diagnosis and treatment is required to decrease fatality. Combination of amphotericin-B and flucytosine is the recommended initial treatment for the first two weeks. Fluconazole is started after two weeks of combination therapy. ${ }^{[7-9]}$

Mortality from developed countries, secondary to HIVassociated cryptococcal meningitis is in the range of 10$30 \%$ while in developing countries the mortality rate is substantial, around $13-40 \%$ because of late presentation, limited access to antifungal therapy, and inability to adequately monitor intracranial pressure among many other reasons. ${ }^{[7]}$ Since there is little information specifically addressing cryptococcal meningitis in HIVinfected patients from the Indian subcontinent, we carried out this analysis. 


\section{Materials and Methods}

This retrospective observational study was carried out at the Post Graduate Institute of Medical Education and Research (PGIMER) Chandigarh, a tertiary care referral hospital in Northern India. The catchment area of this hospital includes Chandigarh union territory and the adjacent states of Himachal Pradesh, Punjab, Haryana, Uttrakhand, Uttar Pradesh and Jammu-Kashmir. Patients presenting with cryptococcal meningitis between January 2005 and March 2008 were studied. Cryptococcal meningitis was defined as clinical features of meningitis/meningoencephalitis along with positive cerebrospinal fluid (CSF) cryptococcal antigen test or positive CSF India ink preparation or isolation of Cryptococcus neoformans in the CSF culture. Patients presenting with clinical features of meningitis/meningoencephalitis of less than four weeks duration were defined as having acute or subacute presentation.

Patient's demographic data, risk factors for acquisition of HIV infection, prior acquired immune deficiency syndrome (AIDS) defining conditions, other concurrent infections, presenting clinical symptomatology, physical findings, laboratory parameters, CSF examination findings, side-effects of treatment, development of any complications and hospital outcome were analyzed. All patients received amphotericin-B (0.7 mg/kg/day) for two weeks. Flucytosine was not given as it was not available. Fluconazole ( $400 \mathrm{mg} /$ day) was given for the next six weeks. Subsequently, secondary prophylaxis with fluconazole $(200 \mathrm{mg} /$ day) was continued till the CD4 count had increased to more than $200 / \mathrm{mm}^{3}$ for $\geq$ six months. Follow-up data of these patients after discharge was recorded. During follow-up any recurrence of cryptococcal meningitis, possible reasons of recurrence, type of treatment received, complications developed and outcome was recorded as well.

Various laboratory abnormalities were defined using cutoff values as follows: anemia - hemoglobin $<10 \mathrm{~g} /$ dL; leucopenia - TLC $<4 \times 10^{9} / \mathrm{L}$; thrombocytopenia - platelet count $<150 \times 10^{9} / \mathrm{L}$; hypoalbuminaemia albumin $<3.5 \mathrm{~g} / \mathrm{dL}$; hyponatremia- plasma sodium $<$ $135 \mathrm{mEq} / \mathrm{l}$ hypokalemia - plasma potassium < 3.5 $\mathrm{mEq} / \mathrm{L}$; hypomagnesaemia- plasma magnesium $<1.5$ $\mathrm{mg} / \mathrm{dL}$; impaired renal function- blood urea nitrogen $>$ $50 \mathrm{mg} / \mathrm{dL}$ and/or serum creatinine $>1.2 \mathrm{mg} / \mathrm{dL}$; elevated liver enzymes $->2$ times the upper limits of normal [aspartate aminotransferase (AST) - >100 IU/L, alanine aminotransferase (ALT) - >100 IU/L].

The following criteria were used to define common concomitant infections: Pulmonary tuberculosis (PTB): clinical features suggestive of TB with radiological features compatible with TB on chest radiograph or computed tomographic (CT) scan and/or demonstration of acid-fast bacilli (AFB) in sputum smears or growth of Mycobacterium tuberculosis in sputum culture. Disseminated tuberculosis (DTB): clinical features suggestive of $\mathrm{TB}$ with concurrent involvement of at least two non-contiguous organs, in the presence of bacteriological and/or histopathological evidence of TB and improvement with anti-tuberculosis therapy. Pneumocystis jiroveci pneumonia (PCP): bilateral, diffuse interstitial infiltrates on chest radiograph or high-resolution CT, with hypoxemia $\left(\mathrm{PaO}_{2}<12 \mathrm{kPa}\right)$ and sputum smears/cultures negative for aerobic bacteria and AFB and/or demonstration of Pneumocystis jiroveci in induced sputum.

\section{Statistical analysis}

Statistical analysis was done using the statistical software 'SPSS version 13.0' (SPPS Corp, Chicago, IL, USA). Descriptive analysis consisted of mean with standard deviation and range for various parameters. Frequency of various clinical and laboratory findings and the frequencies of individual opportunistic infections are expressed as percentage.

\section{Results}

Forty patients diagnosed to have cryptococcal meningitis were analyzed. These included 35 males. Mean age of these patients was $40.0 \pm 9.1$ years. Most patients were in the age group of 25-49 years. Thirtysix $(90 \%)$ patients presented with headache. Duration of headache ranged from two to 365 days. Fifteen patients had severe headache, while 12 patients had moderate and nine had mild severity of headache. Thirty-one (77.5\%) patients had history of fever during presentation. Duration of fever ranged from one to 730 days. Twenty-two (55\%) patients had acute/ subacute presentation of cryptococcal meningitis. Eighteen (45\%) patients had altered sensorium. Duration of alteration of sensorium ranged from one to 15 days. Focal neurological deficit occurred in three patients. Eleven patients had history of seizures. All of them had generalized tonic-clonic seizures. Detailed clinical symptoms and signs of these patients during the time of presentation are shown in Table 1.

Twenty-one (52.5\%) patients were from the high risk groups including truck drivers and intravenous (IV) drug abuser. An identifiable risk factor for HIV infection was present in 21 (52.5\%) patients. The commonest mode of transmission was multiple heterosexual unprotected sexual contacts, seen in 19 patients. One patient each had history of unsafe blood transfusion and intravenous drug abuse.

Detailed baseline hematological parameters are shown in Table 2. CT scan head was done in all patients. Four patients had features to suggest communicating hydrocephalus, two patients had cerebral edema. One CT scan showed features of cerebral atrophy. Bilateral 


\begin{tabular}{|c|c|c|}
\hline & & \\
\hline Symptoms /signs & Frequency & Percentage \\
\hline Headache & 36 & 90 \\
\hline Fever & 31 & 77.5 \\
\hline Vomiting & 29 & 72.5 \\
\hline Weight loss & 28 & 70 \\
\hline Anorexia & 28 & 70 \\
\hline Altered sensorium & 18 & 45 \\
\hline Seizures & 11 & 27.5 \\
\hline Diarrhea & 7 & 17.5 \\
\hline Cough & 4 & 10 \\
\hline Focal neurological deficits & 3 & 7.5 \\
\hline Photophobia & 3 & 7.5 \\
\hline Blurring of vision & 2 & 5 \\
\hline Glasgow coma scale & $13.64 \pm 2.81$ & \\
\hline Nuchal rigidity & 13 & 32.5 \\
\hline Hepatomegaly & 9 & 22.5 \\
\hline Splenomegaly & 9 & 22.5 \\
\hline Lymphadenopathy & 7 & 17.5 \\
\hline
\end{tabular}

basal ganglia hypodensity, left basal ganglia hypodensity and ring-like enhancing lesion in left frontoparietal region was seen in one patient each.

Twenty (50\%) patients had no cells in CSF examination. Lymphocytic predominance was seen in $55 \%$ of the remaining 20 patients. The rest of the $45 \%$ patients had predominant polymorphonuclear response. Hypoglycorrhchia was seen in 30 (75\%) patients. Eighteen patients had elevated CSF protein. India ink was positive in 34 patients. Cryptococcal antigen test in CSF was positive in all patients. Thirty-six (90\%) patients had CSF culture positive for Cryptococcus neoformans.

The CD4 cell count ranged from 16 to 299 cells $/ \mu$ l. Median CD4 cell count of the whole group was 109 cells/ $\mu \mathrm{l}$. Cryptococcal meningitis was the first AIDS-defining illness in 30 (75\%) patients. Other infective conditions concurrently seen in 21 patients have been shown in detail in Table 3. Twelve patients had past history of infections which included TB in 10 patients, herpes zoster in two and cytomegalovirus (CMV) retinitis in one patient. Out of 17 patients receiving antiretroviral therapy (ART), 14 were receiving it at presentation. It was initiated within the last six months of index presentation in four of these patients. The remaining three were started on ART at the completion of two weeks of amphotericin-B. Six patients were receiving a combination of lamivudine, stavudine and nevirapine. Another six patients were receiving stavudine, lamivudine and efaviranz. Zidovudine, lamivudine and nevirapine combination was prescribed in three and two patients were receiving combination of zidovudine, lamivudine and efaviranz. Duration of ART in those patients already on treatment at the time of presentation ranged from 14 days to six years.

All patients received conventional amphotericin-B $(0.7 \mathrm{mg} / \mathrm{kg} /$ day) for initial two weeks and then were started on fluconazole ( $400 \mathrm{mg} /$ day). Thirty-five patients

\begin{tabular}{|c|c|c|c|c|}
\hline Variable & Unit & Mean $\pm S D$ & Range & $\begin{array}{c}\text { Abnormality } \\
(\%)\end{array}$ \\
\hline Hemoglobin & $\mathrm{g} / \mathrm{dL}$ & $10.3 \pm 2.4$ & $5.0-16.3$ & 47.5 \\
\hline TLC ${ }^{*}$ & X109/L & $6.7 \pm 3.2$ & $1.3-14.8$ & 17.5 \\
\hline Platelet count & $\mathrm{X} 10^{9} / \mathrm{L}$ & $170.9 \pm 99.1$ & $13-401$ & 47.5 \\
\hline CD4 count & $(/ \mu \mathrm{l})$ & $119.3 \pm 66.9$ & 16-299 & 100 \\
\hline
\end{tabular}

${ }^{*} \mathrm{TLC}=$ Total leukocyte count

Table 3: Concurrent opportunistic infections and HIV-related conditions

\begin{tabular}{lcc}
\hline Diagnosis & Frequency & Percentage \\
Oro-pharyngeal candidiasis & 16 & 40 \\
Tuberculosis & 6 & 15 \\
Pulmonary & 3 & 7.5 \\
Extra pulmonary & 3 & 7.5 \\
Diarrhea & 7 & 17.5 \\
Pneumocystis jiroveci pneumonia & 3 & 7.5 \\
Herpes genital ulcer & 2 & 5 \\
Urinary tract infection & 1 & 2.5 \\
Hospital-acquired pneumonia & 2 & 5 \\
\hline
\end{tabular}

developed some adverse effects to amphotericin-B. Commonest side-effect was hypokalemia seen in 29 patients. Acute febrile reaction occurred in 19 patients despite premedication with paracetamol and antihistaminics. Increase in urea and creatinine was seen in 11 patients. Hypomagnesaemia was seen in six patients, hyponatremia in five and anemia in four patients.

Out of 40 patients, 33 patients improved with treatment while three patients died. Four patients left against medical advice and in all of these patients progressive deterioration in various clinical and biochemical parameters were seen during hospital stay. All three patients who died had objective evidence of raised intracranial tension. One of them also had altered sensorium at presentation. Four patients who left against medical advice had alteration of sensorium at presentation which continued to progress during hospital stay despite amphotericin- B therapy and other supportive measurements. Two of them had objective evidence of raised intracranial tension in the form of papilledema on fundus examination and diffuse cerebral edema on CT head and two of them also acquired hospital infection leading to progressive worsening of condition. Patients who improved received consolidation therapy with high-dose fluconazole (400 $\mathrm{mg} /$ day) for the next six weeks. Duration of follow-up in these patients ranged from 21 to 800 days. Mean duration of follow-up was $364.8 \pm 234.1$ days. Fifty per cent patients had a follow-up of more than one year duration. Mean number of visits per patients were $5.9 \pm$ 4.2. All patients who were not receiving ART previously were started on ART four weeks after completion of two weeks amphotericin-B induction therapy. Four patients had recurrence of cryptococcal meningitis. All these 
patients had recurrence of cryptococcal meningitis within six months of the first episode. Non-compliance of fluconazole therapy was the reason for recurrence in all these patients. All except one patient responded to repeat therapy with amphotericin-B.

\section{Discussion}

Most of the patients were in the age group 25-49 years and males dominated the study group. This is in consonance with the technical report providing national level statistics published by National AIDS Control Organization (NACO) in the year 2006. ${ }^{[2]}$ Triad of headache, fever and vomiting were predominant symptoms, similar to the earlier reports in the literature. ${ }^{[7,8,10,11]}$ Cryptococcal meningitis usually presents in the form of chronic meningitis. In this series more than half the patients had acute to subacute presentation. Our findings emphasize that even with acute presentation with features of meningitis in HIV-infected individuals, possibility of cryptococcal meningitis must always be considered and patients must be investigated accordingly. Alteration of sensorium was seen in $45 \%$ patients as compared with other studies showing this in 13-73\% of patients. ${ }^{[10,11-18]}$ Seizures have been reported in 9-35\% of these patients. ${ }^{[10,11,18]}$ In this study, $27.5 \%$ of patients had seizures. Focal neurological deficit was seen in a small number of patients, which is in consonance with other case series. ${ }^{[10-11]}$

Commonest associated infections were oral candidiasis and TB which are in consonance with two large studies on HIV-infected patients reported from the Indian subcontinent. ${ }^{[3,4]}$ In a significant percentage of patients in this case series, cryptococcal meningitis was the initial AIDS-defining illness which is comparable to that seen in South African and Zambian studies. ${ }^{[11,17,19,20]}$

An identifiable risk factor for HIV infection was seen in half of study group. Heterosexual mode was the commonest mode of transmission of HIV infection. The possible reasons for not revealing the source of infection or high-risk behavior could be prevailing social values which forbid any sexual relationship other than heterosexual mode, not wanting to disclose his/ her personal information etc.

Due to the immunocompromised status, CSF has high load of cryptococcal organisms and less of inflammatory response leading to lower white cell counts in HIV-infected patients. ${ }^{[10,11,13,14]}$ Thus, India ink is positive in $70-90 \%$ of AIDS patients and in only $50 \%$ of non-AIDS patients. ${ }^{[7,8]}$ In our patients, 50\% (20) patients had no white blood cell in CSF and 85\% (35) patients had India ink test positive. Hypoglycorrhchia was seen in $75 \%$ of patients. Detection of cryptococcal capsular polysaccharide antigen is an important test in diagnosing this infection. Various studies have shown overall sensitivities and specificities of this test in the range of $93-100 \%$ and $93-98 \%$ respectively. False positive results of this test are approximately $0-0.4 \%{ }^{[7,8]}$ All our patients had positive cryptococcal antigen test done in CSF.

The CD4+ T cell count is the best indicator of the immediate state of immunologic competence and also the strongest predictor of HIV-related complications in these patients. Cryptococcal infection was the major opportunistic infection and a major cause of death in HIV-infected patients with CD4 cell count $<100$ cells/ $\mu$ l in the pre-highly active antiretroviral therapy era. ${ }^{[8]}$ In one study the average CD4 lymphocyte count in patients with AIDS and cryptococcosis was 73 cells/ $\mu l .{ }^{[21]}$ Median CD4 cell count in this case series was 109 cells/ $\mu$ l.

The immune reconstitution inflammatory syndrome (IRIS) is a spectrum of clinical signs and symptoms resulting from the restored ability to mount an inflammatory response associated with immune recovery. The incidence of IRIS was $10-32 \%$ in various studies on HIV patients being initiated on ART. ${ }^{[22-24]}$ Clinical events occurring before the first six months of ART therapy often represent IRIS related to preexisting conditions. In this series, out of 14 patients receiving ART at presentation, four patients receiving ART for six months or less presented with features of cryptococcal meningitis possibly indicating IRIS. Median CD4 cell count of these patients was considerably lower (79 cells $/ \mu \mathrm{l}$ ) as compared to the whole group (109 cells/ $\mu \mathrm{l}$ ). The remaining 10 patients were receiving ART for more than six months. The development of a new or recurrent World Health Organization (WHO) Stage 3 or 4 condition after the first six months of ART is considered functional evidence of HIV disease progression. This finding of the present study has important implications and these patients developing opportunistic infection after six months of ART should be the focus of investigations to exactly pinpoint cause of treatment failure, which because of the retrospective nature of this study was not feasible.

Untreated cryptococcal meningitis is uniformly fatal. A large randomized controlled trial undertaken by Mycoses Study Group established the combination of amphotericin-B deoxycholate $(0.7 \mathrm{mg} / \mathrm{kg} /$ day $)$ and flucytosine $(100 \mathrm{mg} / \mathrm{kg} /$ day $)$ as a standard of care for the treatment of cryptococcal meningitis in AIDS patients. ${ }^{[25]}$ The findings of this study were further substantiated by another study. ${ }^{[26]}$ In this case series, due to unavailability of flucytosine, amphotericin-B $(0.7 \mathrm{mg} / \mathrm{kg} /$ day) was used alone.

In a substantial proportion of patients (87.5\%), some adverse effects due to amphotericin- B were noted. These side-effects were managed with premedication, adequate hydration and electrolyte replenishment and were easily treated. None of them was life-threatening. All the patients in this study group ultimately received complete therapy with amphotericin-B. 
In-hospital mortality in this case series was 7.5\%. Out of the patients followed up, one patient died during the initial 10 weeks of follow-up. There was no recurrence of cryptococcal meningitis after six months of follow-up. The National Institute of Allergy and Infectious Diseases Mycoses study group and AIDS clinical trials group showed 5.5\% mortality at the end of two weeks and 9.4\% mortality at the end of 10 weeks with combination therapy of amphotericin-B and flucytosine. ${ }^{[25]}$ In another study, mortality was $14 \%$ at two weeks and $22 \%$ at 10 weeks. $^{[26]}$ One study from Thailand showed $16 \%$ mortality at two weeks and $24 \%$ mortality at four weeks in patients who received high-dose amphotericin-B only for two weeks followed by oral azole therapy. ${ }^{[27]}$ From South African countries, in most of the studies only fluconazole had been used as monotherapy for cryptococcal meningitis and mortality in these studies is disappointingly high. ${ }^{[11,17]}$

The poor prognostic factors are depressed level of consciousness, signs of raised intracranial pressure, depressed CSF cell counts and glucose levels and CSF cryptococcal antigen titer $>1024 .{ }^{[28]}$ The most important of all the poor prognostic factors is the CSF opening pressure with values $\geq 250 \mathrm{~mm} \mathrm{H}_{2} \mathrm{O} \cdot{ }^{[29]} \mathrm{Our}$ observations reemphasize the prognostic importance of alteration of sensorium and raised intracranial tension in these patients as the majority of patients having poor outcome had these poor prognostic factors.

In conclusion, this study demonstrates acute and/or subacute presentation of cryptococcal meningitis in more than half of HIV-infected individuals. In $75 \%$ of patients cryptococcal meningitis was the initial AIDSdefining illness. Four patients receiving ART for six months or less presented with features of cryptococcal meningitis possibly indicating IRIS. Ten patients receiving ART for more than six months had developed cryptococcal meningitis indicating failure of current ART regime. CSF examination besides showing poor inflammatory response had hypoglycorrhchia in $75 \%$ of patients possibly indicating higher organism load. High-dose 'amphotericin-B Only' induction therapy followed by fluconazole consolidation therapy yielded acceptable results. High-dose amphotericin-B resulted in reversible side-effects in a substantial proportion of patients and in none of them these were life-threatening. Alteration of sensorium and raised intracranial tension are poor prognostic factors in these patients. Recurrence of cryptococcal meningitis was seen in the initial six months of follow-up and noncompliance of medication was the reason for recurrence in all of these patients.

\section{References}

1. Simoes EA, Babu GP, John T.J, Nirmala S, Solomon S, Lakshminarayana CS, et al. Evidence for HTLV-3 infection in prostitutes in Tamilnadu (India). Indian J Med Res 1987;85:335-8.
2. National AIDS Control Organization. Ministry of Health and Family Welfare, Government of India: Technical report India HIV estimates-2006.

3. Kumarasamy N, Solomon S, Flanigan TP, Hemalatha R, Thyagarajan SP, Mayer KH. Natural history of human immunodeficiency virus disease in southern India. Clin Infect Dis 2003;36:79-85.

4. Sobhani R, Basavaraj A, Gupta A, Bhave AS, Kadam DB, Sangle SA, et al. Mortality \& clinical characteristies of hospitalized adult patients with HIV in Pune, India. Indian J Med Res 2007;126:116-21.

5. Kumarasamy N, Vallabhaneni S, Flanigan TP, Mayer KH, Solomon S. Clinical profile of HIV in India. Indian J Med Res 2005;121:377-94.

6. Sharma SK, Kadhiravan T, Banga A, Goyal T, Bhatia I, Saha PK. Spectrum of clinical disease in a series of 135 hospitalised HIV-infected patients from north India. BMC Infect Dis 2004;4:52.

7. Bicanic T, Harrison TS: Cryptococcal meningitis. Br Med Bull 2005;72:99-118.

8. Perfect JR, Casadevall A. Cryptococeosis. Infect Dis Clin North Am $2002 ; 16: 837-74$

9. Saag MS, Graybill RJ, Larsen RA, Pappas PG, Perfect JR, Powderly WG, et al. Practice guidelines for the management of cryptococcal disease: Infectious Diseases Society of America. Clin Infect Dis 2000;30:710-8

10. Satishchandra P, Mathew T, Gadre G, Nagarathna S, Chandramukhi A, Mahadevan A, et al. Cryptococcal meningitis: Clinical, diagnostic and therapeutic overviews. Neurol India 2007;55:226-32.

11. Mwaba P, Mwansa J, Chintu C, Pobee J, Scarborough M, Portsmouth $\mathrm{S}$, et al. Clinical presentation, natural history, and cumulative death rates of 230 adults with primary cryptococeal meningitis in Zambian AIDS patients treated under local conditions. Postgrad Med J 2001;77:769-73.

12. Satishchandra P, Nalini A, Gourie-Devi M, Khanna N, Santosh V, Ravi V, et al. Profile of neurologic disorders associated with HIV/ AIDS from Bangalore, south India (1989-96). Indian J Med Res $2000 ; 111: 14-23$.

13. Khanna N, Chandramuki A, Desai A, Ravi V. Cryptococeal infection of the central nervous system: an analysis of predisposing factors, laboratory findings and outcome in patients from south India with special reference to HIV infection. J Med Microbiol 1996;45:376-9.

14. Kalra SP, Chadha DS, Singh AP, Sanchetee PC, Mohapatra AK. Cryptococeal meningitis in acquired immunodeficiency syndrome. J Assoc Physicians India 1999;47:958-61.

15. Lakshmi V, Sudha T, Teja VD, Umabala P. Prevalence of central nervous system cryptococeosis in human immunodeficiency virus reactive hospitalized patients. Indian J Med Microbiol 2007;25:146-9.

16. Prasad KN, Agarwal J, Nag VL, Verma AK, Dixit AK, Ayyagari A. Cryptococcal infection in patients with clinically diagnosed meningitis in a tertiary care center. Neurol India 2003;51:364-6.

17. Schaars CF, Meintjes GA, Morroni C, Post FA, Maartens G. Outcome of AIDS-associated cryptococcal meningitis initially treated with $200 \mathrm{mg} /$ day or $400 \mathrm{mg}$ /day of fluconazole. BMC Infect Dis 2006;6:118.

18. Kisenge PR, Hawkins AT, Maro VP, McHele JP, Swai NS, Mueller A, et al. Low CD4 count plus coma predicts cryptococcal meningitis in Tanzania. BMC Infect Dis 2007:7:39.

19. Heyderman RS, Gangaidzo IT, Hakim JG, Mielke J, Taziwa A, Musvaire P, et al. Cryptococcal meningitis in human immunodeficiency virus-infected patients in Harare, Zimbabwe. Clin Infect Dis 1998;26:284-9.

20. Moosa MY, Coovadia YM. Cryptococeal meningitis in Durban, South Africa: a comparison of clinical features, laboratory findings and outcome for human immunodeficiency virus (HIV) -positive and HIVnegative patients. Clin Infect Dis 1997;24:131-4.

21. Crowe SM, Carlin JB, Stewart KI, Lucas CR, Hoy JF. Predictive value of CD4 lymphocyte numbers for the development of opportunistic infections and malignancies in HIV-infected persons. J Acquir Immune Defic Syndr 1991;4:770-6.

22. Murdoch DM, Venter WD, Feldman C, Van Rie A. Incidence and risk factors for the immune reconstitution inflammatory syndrome in HIV patients in South Africa: a prospective study. AIDS 2008;22:601-10.

23. Shelburne SA, Visnegarwala F, Darcourt J, Graviss EA, Giordano TP, White AC Jr, et al. Incidence and risk factors for immune reconstitution 
inflammatory syndrome during highly active antiretroviral therapy. AIDS 2005;19:399-406.

24. Ratnam I, Chiu C, Kandala NB, Easterbrook PJ. Incidence and risk factors for immune reconstitution inflammatory syndrome in an ethnically diverse HIV type 1-infected cohort. Clin Infect Dis 2006;42:418-27.

25. Van der Horst CM, Saag MS, Cloud GA, Hamill RJ, Graybill JR, Sobel JD, et al. Treatment of cryptococcal meningitis associated with the acquired immunodeficiency syndrome: National Institute of Allergy and Infectious Diseases Mycoses Study Group and AIDS Clinical Trials Group. N Engl J Med 1997;337:15-21.

26. Brouwer AE, Rajanuwong A, Chierakul W, Griffin GE, Larsen RA, White NJ, et al. Combination antifungal therapies for HIVassociated cryptococcal meningitis: A randomised trial. Lancet 2004;363:1764-7.
27. Pitisuttithum P, Tansuphasawadikul S, Simpson AJ, Howe PA, White NJ. A prospective study of AIDS-associated cryptococcal meningitis in Thailand treated with high-dose amphotericin B. J Infect 2001;43:226-33.

28. Diamond RD, Bennett JE. Prognostic factors in cryptococcal meningitis: A study in 111 cases. Ann Intern Med 1974;80:176-81.

29. Graybill JR, Sobel J, Saag M, van Der Horst C, Powderly W, Cloud G, et al. Diagnosis and management of increased intracranial pressure in patients with AIDS and cryptococcal meningitis: The NIAID Mycoses Study Group and AIDS Cooperative Treatment Groups. Clin Infect Dis 2000;30:47-54

Accepted on 26-06-2008

Source of Support: Nil, Conflict of Interest: None declared.

\section{Author Help: Reference checking facility}

The manuscript system (www.journalonweb.com) allows the authors to check and verify the accuracy and style of references. The tool checks the references with PubMed as per a predefined style. Authors are encouraged to use this facility before submitting articles to the journal.

- The style as well as bibliographic elements should be $100 \%$ accurate to get the references verified from the system. A single spelling error or addition of issue number / month of publication will lead to error to verifying the reference.

- $\quad$ Example of a correct style

Sheahan P, O'leary G, Lee G, Fitzgibbon J. Cystic cervical metastases: Incidence and diagnosis using fine needle aspiration biopsy. Otolaryngol Head Neck Surg 2002;127:294-8.

- Only the references from journals indexed in PubMed would be checked.

- Enter each reference in new line, without a serial number.

- $\quad$ Add up to a maximum 15 reference at time.

- If the reference is correct for its bibliographic elements and punctuations, it will be shown as CORRECT and a link to the correct article in PubMed will be given.

- If any of the bibliographic elements are missing, incorrect or extra (such as issue number), it will be shown as INCORRECT and link to possible articles in PubMed will be given. 